\title{
ORIGINAL ARTICLE \\ Bile salt hydrolase-overexpressing Lactobacillus strains can improve hepatic lipid accumulation in vitro in an NAFLD cell model
}

\author{
Wenli Huang ${ }^{\dagger}$, Guangqiang Wang ${ }^{\dagger}$,Yongjun Xia, Zhiqiang Xiong and Lianzhong Ai* \\ Shanghai Engineering Research Center of Food Microbiology, School of Medical Instrument and Food Engineering, \\ University of Shanghai for Science and Technology, Shanghai, China
}

\begin{abstract}
Popular scientific summary
Lactobacillus plantarum AR113 and Lactobacillus casei pWQH01 with high bile salt hydrolase active can improve steatosis by reducing the expression of SREBP-1c, ACC, FAS and TNF- $\alpha$ and increasing the expression of AMPK and PPAR $\alpha$ in vitro.
\end{abstract}

\section{Abstract}

Background: Non-alcoholic fatty liver disease (NAFLD) includes a range of liver diseases that occur in the absence of significant alcohol consumption. The probiotic bacterial strains Lactobacillus casei LC2W, which overexpresses the bile salt hydrolase (BSH) gene (referred to as pWQH01), and Lactobacillus plantarum AR113, which exhibits high BSH activity, have been shown to improve hepatic lipid accumulation and may lower cholesterol levels in vivo. These effects may be BSH-dependent, as $L$. casei LC2W without BSH activity did not exert these beneficial effects.

Objective: This study aimed to investigate the effects of Lactobacillus with high BSH activity on cholesterol accumulation and lipid metabolism abnormalities in oleic acid (OA)- and cholesterol-induced HepG2 cell models, and to determine the mechanism underlying the effects.

Design: A HepG2 cell model of OA-induced steatosis and cholesterol-induced cholesterol accumulation was developed. OA- and cholesterol-treated HepG2 cells were incubated with L. plantarum AR113, L. casei LC2W or L. casei pWQH01 for $6 \mathrm{~h}$ at $37^{\circ} \mathrm{C}$ with $5 \% \mathrm{CO}_{2}$. Subsequently, a series of indicators and gene expressions were analysed.

Results: Both L. plantarum AR113 and L. casei pWQH01 significantly reduced lipid accumulation, total cholesterol (TC) levels and 3-hydroxy-3-methyl-glutaryl-coenzyme A reductase (HMGCR) mRNA expression relative to the control group, whereas L. casei LC2W had no similar effect. Additionally, exposure to L. plantarum AR113 or L. casei pWQH01 significantly reduced the expression of sterol regulatory element-binding protein 1c (SREBP-1c), Acetyl-CoA carboxylase (ACC), fatty acid synthase (FAS) and tumour necrosis factor- $\alpha(\mathrm{TNF}-\alpha)$ andsignificantly increased the expression of 5 adenosine monophosphate-activated protein kinase (AMPK) and peroxisome proliferator-activated receptor alpha $(\operatorname{PPAR} \alpha)$.

Conclusion: Both L. plantarum AR113 and L. casei pWQH01 appear to improve steatosis in vitro in a BSHdependent manner.

Keywords: Lactobacillus; bile salt hydrolase; non-alcoholic fatty liver disease; hepatic lipid accumulation; HepG2 cell; cholesterol

Received: 2 November 2019; Revised: 23 May 2020; Accepted: 3 June 2020; Published: 12 November 2020

$\mathrm{N}$ on-alcoholic fatty liver disease (NAFLD) is a clinicopathological syndrome characterised by hepatic steatosis at an alcohol consumption level of $<20 \mathrm{~g}$ per day (1). The global incidence of NAFLD continues to increase and thus parallels the increasing prevalence of obesity, hyperlipidaemia, type 2 diabetes and metabolic syndrome (2-4). Several previous studies demonstrated that hepatic steatosis occurs due to

\footnotetext{
†These authors contributed equally.
} 
increased lipogenesis and reduced lipid export from the liver. Therefore, the hepatic accumulation of neutral lipids derived from an imbalance in lipid acquisition and clearance is a hallmark of NAFLD. During NAFLD, hepatocytes exhibit altered cholesterol metabolism, which is characterised by the activation of cholesterol biosynthesis pathways, enhancement of cholesterol desertification and reduction in the cholesterol output and bile acid synthesis $(5,6)$. Currently, NAFLD treatment is limited to a restricted energy intake, increased physical activity and pharmacological treatments. However, these interventions often have unsatisfactory effects (7). The pathogenesis of NAFLD is often referred to as a 'two hit' pathophysiological theory. The 'first hit' is the deposition of free fatty acids (FFAs) in hepatocytes (i.e. steatosis), while the 'second hit' is the progression of steatosis to nonalcoholic steatohepatitis (NASH) (8). This progression is often associated with the release of cytokines, and a previous study identified a central role for the overexpression of pro-inflammatory cytokine tumour necrosis factor (TNF)- $\alpha$ mRNA in the aetiology of NASH (9). Human liver steatosis is associated with the accumulation of excess oleic acid (OA), a monosaturated omega-9 fatty acid and end product of de novo fatty acid synthesis (10). Morphologically, OA-treated human hepatoblastoma HepG2 cells and steatotic hepatocytes are similar (11).

Probiotics are the most widely used enteric microecological preparations in clinical settings. These microbes colonise the intestinal and reproductive systems of the body and can promote the microecological balance in the host, thereby improving disease symptoms and promoting health (12). Internationally, effective and complete probiotic strains must conform to the following four criteria: (1) beneficial to the host, (2) normal colonisation at a specific location in the host, (3) growth and survival at a specific location in/on the host and (4) maintenance of viability and activity during production and storage (13). Commonly used probiotics can be classified as Lactobacillus, Bifidobacterium, Gram-positive cocci and some fungi (14). The 2015 Recommended Guide for Probiotics Application identified the following probiotic preparations as preferred for the treatment of NAFLD: Lactobacillus plantarum, Lactobacillus rhamnosus, Bifidobacterium bifidum, Streptococcus thermophilus and Bifidobacterium longum. Many studies have shown that probiotics can improve liver function, blood lipid profiles, insulin resistance, inflammatory factors and even the degree of liver fibrosis in NAFLD patients, indicating that these agents may have potential therapeutic value in this population $(15,16)$. Recent studies have demonstrated that bile acids can significantly impact many disease states, including NAFLD $(16,17)$. The gut microbiota may affect bile acid metabolism by modulating a critical step catalysed by bile salt hydrolases (BSHs), which hydrolyse the amide bond and release glycine or taurine from the steroid cores of primary bile acids. The forming deconjugated bile acids can undergo different subsequent transformations that may affect the physiology and disease status of the host (17), so many researchers speculate that BSH may play an important role. BSH is an important gatekeeper to subsequent bile acid transformations, but it remains unclear whether high BSH activity can relieve NAFLD and if so, by which mechanism.

In our previous work, we observed that $L$. plantarum AR113 (high BSH activity) and L. casei pWQH01 which overexpresses BSH from L. plantarum AR113 (high BSH activity) ameliorated cholesterol accumulation in vivo, whereas $L$. casei LC2W (no BSH activity) had no obvious effect on hypercholesterolemia (18). This study aimed to investigate the beneficial effects of Lactobacillus strains with high BSH activity on cholesterol accumulation and lipid metabolism abnormalities in vitro, using OA- and cholesterol-induced HepG2 cells as a model for NAFLD, and to subsequently determine the underlying mechanism.

\section{Materials and methods}

\section{Bacterial strains, growth conditions and treatments}

L. plantarum AR113, L. casei $\mathrm{pWQH} 01$ and $L$. case $\mathrm{LC} 2 \mathrm{~W}$ strains were obtained from the Shanghai Engineering Research Centre of Food Microbiology, University of Shanghai for Science and Technology (Shanghai, China). L. casei $\mathrm{LC} 2 \mathrm{~W}$ does not harbour the $b$ shl gene and thus lacks BSH activity. L. casei $\mathrm{pWQH} 01$ overexpresses bshl, and this gene is also responsible for the high level of BSH activity in L. plantarum AR113. The AR113, pWQH01 and LC2W strains were stored at $-80^{\circ} \mathrm{C}$ in de Man, Rogosa and Sharpe (MRS) broth containing 20\% glycerol. The strains were activated three successive times in sterile MRS broth (Difco, Detroit, MI, USA) and incubated at $37^{\circ} \mathrm{C}$ for $24 \mathrm{~h}$. Prior to experimental use, a $2 \%(\mathrm{v} / \mathrm{v})$ inoculum of each strain was sub-cultured and incubated at $37^{\circ} \mathrm{C}$ for $24 \mathrm{~h}$. Each probiotic suspension was adjusted to a concentration of $1 \times$ $10^{9} \mathrm{CFU} / \mathrm{mL}$. Next, the probiotic suspensions were centrifuged at 4,000 rpm for $5 \mathrm{~min}$, and the pellets were washed with phosphate buffered saline (PBS) and resuspended in non-antibiotic supplemented cell culture medium.

\section{Oleic acid/BSA complex solution preparation}

An oleic acid/Bovine serum albumin (OA/BSA) complex solution was prepared as previously described, with minor modifications (19). First, a $100 \mathrm{mM}$ OA stock solution was prepared in $0.1 \mathrm{~N} \mathrm{NaOH}$ by heating to $70^{\circ} \mathrm{C}$ in a shaking water bath. Next, a $10 \%(\mathrm{w} / \mathrm{v})$ BSA solution was prepared in $\mathrm{H}_{2} \mathrm{O}$ by heating to $55^{\circ} \mathrm{C}$ in a shaking water bath. Finally, a $10 \mathrm{mM}$ OA solution containing $10 \%$ BSA was diluted in culture medium to obtain the desired final concentrations. The OA/BSA complex solution was sterile-filtered through a $0.45-\mu \mathrm{m}$ membrane filter and stored at $-20^{\circ} \mathrm{C}$. 


\section{Cell culture and treatment}

The human hepatocellular liver carcinoma cell line, HepG2, was purchased from the Cell Resources Centre of Shanghai Institutes for Biological Sciences (Shanghai, China). Briefly, cells were cultured in Dulbecco's Modified Eagle Medium (DMEM) containing 10\% fetal bovine serum (FBS), $100 \mathrm{U} /$ $\mathrm{mL}$ penicillin and $100 \mathrm{U} / \mathrm{mL}$ streptomycin in an incubator at $37^{\circ} \mathrm{C}$ under a humidified atmosphere of air containing $5 \% \mathrm{CO}_{2}$ until an $\sim 80 \%$ confluent monolayer was obtained. All cell culture components were purchased from Gibco ${ }^{\mathrm{TM}}$ (Thermo Fisher Scientific, Grand Island, NY, USA). HepG2 monolayers were pre-incubated with non-antibiotic supplemented cell culture medium for $6 \mathrm{~h}$ before further assays. A HepG2 cell model of OA-induced steatosis and cholesterol-induced cholesterol accumulation was developed (20). Cells were seeded at a density of $5 \times 10^{5}$ cells $/ \mathrm{mL}$ in six-well plates and cultured in FBS-free medium for $24 \mathrm{~h}$ in triplicate, after which the cells were induced by exposure to $1 \mathrm{mM}$ OA and $75 \mu \mathrm{g} / \mathrm{mL}$ water-soluble cholesterol (Sigma-Aldrich, Saint Louis, MO, USA) for $6 \mathrm{~h}$. Control group cells were treated with OA-free and cholesterol-free medium containing $1 \%$ BSA. Finally, $1 \mathrm{~mL}$ of each probiotic suspension was added to the OA- and cholesterol-treated HepG2 cells, which were incubated for $6 \mathrm{~h}$ at $37^{\circ} \mathrm{C}$ with $5 \% \mathrm{CO}_{2}$. A series of experimental analyses were performed as described below.

\section{Cell viability assay}

To assess cell viability, $5 \times 10^{4}$ HepG 2 cells in $100 \mu \mathrm{L}$ were seeded in 96-well plates and cultured overnight. Next, the HepG2 cells were treated with L. plantarum AR113, L. casei LC2W or L. casei pWQH01 and a mixture of $1 \mathrm{mM} \mathrm{OA}$ with $50 \mu \mathrm{g} / \mathrm{mL}$ cholesterol, $1 \mathrm{mM}$ OA with $75 \mu \mathrm{g} / \mathrm{mL}$ cholesterol or $1 \mathrm{mM}$ OA with $100 \mu \mathrm{g} / \mathrm{mL}$ cholesterol for 2, 4, 6 or $8 \mathrm{~h}$ under the same culture conditions described above. Finally, cell viability was measured using a Cell Counting Kit-8 (CCK-8) assay kit (Beyotime, Shanghai, China) as per the manufacturer's instructions $(n=3)$.

\section{Oil Red O staining of HepG2 cells}

After treatment with each of the probiotic suspensions for 6 h, HepG2 cells were carefully washed three times with cold PBS to avoid disruption and subsequently fixed with $10 \%$ formalin solution for $20 \mathrm{~min}$ at room temperature. The fixed cells were then stained with a freshly prepared working solution of Oil Red O (Nanjing Jianchen Bioengineering Institute, Jiangsu, China) in the dark for $15 \mathrm{~min}$. Next, the stained cells were washed several times with cold distilled water and observed and imaged under an inverted microscope (Nikon, Tokyo, Japan). The intracellular lipid-bound stain was re-dissolved in 100\% isopropanol and shaken at room temperature for $10 \mathrm{~min}$, after which the resultant solution was transferred to a 96 -well plate. The optical density of the resultant solution was read at a wavelength of $510 \mathrm{~nm}$.

\section{Intracellular cholesterol content of HepG2 cells}

After exposing the cells to L. plantarum AR113, L. casei LC2W or L. casei pWQH01 for $6 \mathrm{~h}$, the culture media was removed carefully without disturbing the cells. The cells were gently washed with cold PBS, lysed in radioimmunoprecipitation assay (RIPA) buffer and homogenised. The homogenate was centrifuged at $13,000 \mathrm{rpm}$ and $4^{\circ} \mathrm{C}$ for 20 min to remove the insoluble materials, after which the intracellular cholesterol content was normalised against the total cellular protein content using a BCA protein assay kit (Beyotime, Shanghai, China).

\section{Cholesterol uptake assay}

Cholesterol uptake was analysed by comparing HepG2 cells treated with the viable probiotic strains, the mixture of OA and cholesterol or no treatment. The cholesterol concentrations in the cells were measured using the modified 0 -pthalaldehyde colorimetric method, as described by Gilliland (21). Briefly, $0.25 \mathrm{~mL}$ of homogenate was mixed with $1.5 \mathrm{~mL}$ of $95 \%(\mathrm{v} / \mathrm{v})$ ethanol and 1 $\mathrm{mL}(50 \%, \mathrm{w} / \mathrm{v})$ of $\mathrm{KOH}$, vortexed for $15 \mathrm{~s}$ and heated at $60^{\circ} \mathrm{C}$ in a water bath for $10 \mathrm{~min}$. After cooling the mixture to room temperature, $2.5 \mathrm{~mL}$ of hexane and $1.5 \mathrm{~mL}$ of distilled water were added and the resultant mixture was vortexed. The hexane layer was transferred into a new glass tube and evaporated under a nitrogen gas flow at $60^{\circ} \mathrm{C}$. Next, $0.75 \mathrm{~mL}$ of 0 -phthalaldehyde reagent $(0.05 \mathrm{~g}$ 0 -pthalaldehyde dissolved in $100 \mathrm{~mL}$ glacial acetic acid) was immediately added to the residue. Finally, $1 \mathrm{~mL}$ of concentrated $\mathrm{H}_{2} \mathrm{SO}_{4}$ was added, and the resultant mixture was mixed thoroughly and incubated at room temperature for a further $10 \mathrm{~min}$ before reading the absorbance at $550 \mathrm{~nm}$. A standard curve was generated using solutions of $0,0.02,0.05,0.10,0.12,0.15$ and $0.20 \mathrm{mg} / \mathrm{mL}$ cholesterol $\left(R^{2}=0.9992\right)$. All the above-listed organic reagents were purchased from the Beijing Land Bridge Technology Co., Ltd. (Beijing, China).

\section{Analysis of lipid-regulating genes in HepG2 cells by qRT-PCR} Following treatment with each of the three probiotic strains for $6 \mathrm{~h}$, the cells were gently washed twice with cold PBS and lysed in RIPA buffer, after which the total RNA was extracted as per the manufacturer's instructions. The amount of RNA was determined, and the purity was checked by measuring the optical densities at 260 and $280 \mathrm{~nm}$. Next, cDNA was synthesised from the isolated total RNA using a cDNA RT PreMix kit (TaKaRa Bio, Otsu, Japan) as per the manufacturer's protocol, and subjected to quantitative reverse transcription polymerase chain reaction (qRT-PCR) using SYBR Premix Ex TaqII (TaKaRa Bio, Otsu, Japan) on a LightCycler ${ }^{\circledR} 96$ (Roche, Basel, Switzerland). PCR amplification was performed using following conditions: $95^{\circ} \mathrm{C}$ for $30 \mathrm{~s}$, followed by 40 cycles of $95^{\circ} \mathrm{C}$ for $5 \mathrm{~s}$ and $60^{\circ} \mathrm{C}$ for $30 \mathrm{~s}$. 
The primer sequences (Sangon Biological Engineering, Shanghai, China) used in this study are shown in Table 1. Gene expression was normalised to the glyceraldehyde 3-phosphate dehydrogenase (GAPDH) mRNA levels. The transcript levels in each group were determined using the $2^{-(\Delta \Delta \mathrm{C} t)}$ method (22) and are expressed as a ratio of expression relative to the control group.

\section{Statistical analyses}

All results are expressed as means \pm standard deviations. Between-group differences were assessed using a one-way

Table 1. Specific human gene primer sequences

\begin{tabular}{|c|c|}
\hline Gene name & Primer sequences \\
\hline \multirow{2}{*}{ GAPDH } & Forward 5'-CGCTCTCTGCTCСTCCTGTT-3' \\
\hline & Reverse 5'-CCATGGTGTCTGAGCGATGT-3' \\
\hline \multirow{2}{*}{ AMPK } & Forward 5'-AGGATGCCTGAAAAGCTTGA -3' \\
\hline & Reverse 5'-GACAGCCGGAGAAGCAGAAAC-3' \\
\hline \multirow{2}{*}{ SREBP-IC } & Forward 5'-GCGGAGCCATGGATTGCAC-3' \\
\hline & Reverse 5'-TCTTCCTTGATACCAGGCCC-3' \\
\hline \multirow{2}{*}{ ACC } & Forward 5'-TGTCTGAAGAGATTAGGGAAGT-3' \\
\hline & Reverse 5'-GTTATGTGAAAGATGTGGATGA-3' \\
\hline \multirow{2}{*}{ FAS } & Forward 5'-TTCGTTTGTGAGCCTGACTGC-3' \\
\hline & Reverse 5'-GCTCCCGGATCACCTTCTTG-3' \\
\hline \multirow{2}{*}{ PPAR $\alpha$} & Forward 5'-TCCGACTCCGTCTTCTTGAT-3' \\
\hline & Reverse 5'-GCCTAAGGAAACCGTTCTGTG-3' \\
\hline \multirow{2}{*}{ HMGCR } & Forward 5'-GACCTTTCCAGAGCAAGCAC-3' \\
\hline & Reverse 5'-TCAACAAGAGCATCGAGGGT-3' \\
\hline \multirow{2}{*}{ TNF $\alpha$} & Forward 5'-CAGCCTCTTCTCCTTCCTGAT-3' \\
\hline & Reverse 5'-GCCAGAGGGCTGATTAGAGA-3' \\
\hline
\end{tabular}

analysis of variance (ANOVA) with Tukey's multiple comparison test. Statistical analyses were performed using the Statistical Package for the Social Sciences (SPSS) statistical software package, version 20 (IBM, Armonk, NY, USA), and a $p$ value $<0.05$ was considered statistically significant.

\section{Results}

Cytotoxic effects of probiotic strains and combined $O A$ and cholesterol treatment on HepG2 cells

Treatment of HepG2 cells with the various probiotic strains (L. plantarum AR113, L. casei LC2W and L. casei pWQH01) for 2, 4, 6 or $8 \mathrm{~h}$ did not cause any cytotoxicity. However, the cell viability was greatly reduced after an 8-h co-incubation, compared to a 6-h co-incubation. Therefore, $6 \mathrm{~h}$ was selected as the endpoint for subsequent experiments.

HepG2 cells were treated with a mixture of $1 \mathrm{mM} \mathrm{OA}$ plus 50,75 or $100 \mu \mathrm{g} / \mathrm{mL}$ cholesterol for $2,4,6$ or $8 \mathrm{~h}$ to induce hepatic steatosis. We estimated the impact of each treatment on cell survival based on the following cell viability criteria $(23,24)$ : $>90 \%$, unaffected; $80-90 \%$, moderately affected and $<80 \%$, cytotoxicity of the compound(s). The mixture of OA and cholesterol suppressed the proliferation of HepG2 cells in a dose-dependent manner (Fig. 1). Cells treated with $1 \mathrm{mM}$ OA plus 50, 75 or $100 \mu \mathrm{g} / \mathrm{mL}$ cholesterol for $6 \mathrm{~h}$ yielded viability values of $118 \%, 93 \%$ and $80 \%$, respectively. The viability of cells treated with $75 \mu \mathrm{g} / \mathrm{mL}$ cholesterol and $1 \mathrm{mM} \mathrm{OA}$ did not differ significantly when compared to the control group. Therefore, $75 \mu \mathrm{g} / \mathrm{mL}$ cholesterol and $1 \mathrm{mM}$ OA were used to induce hepatic steatosis in subsequent experiments.

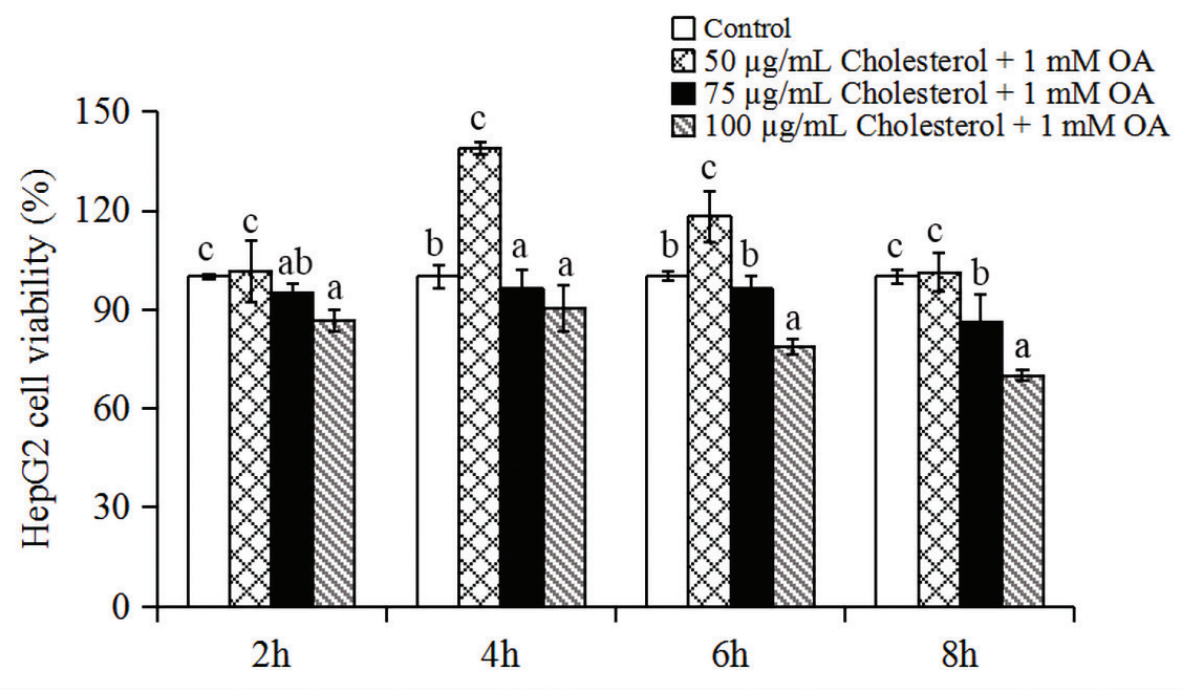

Fig. 1. The effects of different concentrations of cholesterol and $1 \mathrm{mM}$ oleic acid (OA) on the viability of HepG2 cells after treatment for 2, 4, 6 and $8 \mathrm{~h}$. Data represent the means \pm standard deviations $(n=3)$. Statistical differences between groups were determined by a one-way ANOVA. Different letters indicate statistically significant differences between groups, $P<0.05$. 
Probiotic strains with high BSH activity reduced lipid accumulation and lipid content of HepG2 cells

Oil Red $\mathrm{O}$ staining was used to determine the effects of the probiotic strains on cellular lipid accumulation. L. casei $\mathrm{LC} 2 \mathrm{~W}$ and $L$. casei $\mathrm{pWQH} 01$ differ only in terms of BSH activity; the former has no BSH activity, while the latter overexpresses L. plantarum AR113 bsh1 and exhibits high $\mathrm{BSH}$ activity. Oil Red $\mathrm{O}$ staining indicated that HepG2 cells in the control group did not experience significant steatosis, whereas cells in the OA and cholesterol-treated group exhibited severe steatosis. Cells treated with L. plantarum AR113 and L. casei pWQH01 exhibited significantly decreased lipid accumulation relative to the OA- and cholesterol-treated cells. In contrast, L. casei
LC2W treatment did not significantly reduce the intracellular lipid content (Fig. 2a). L. plantarum AR113 and L. casei pWQH01 also significantly decreased the total lipid content relative to the OA- and cholesterol-treated groups, as reflected by a spectrophotometric analysis; again, L. casei LC2W had no significant effect (Fig. 2b).

Probiotic strains with high BSH activity reduced the TC levels in HepG2 cells by attenuating HMGCR expression

Recent studies showed that disordered cholesterol metabolism can lead to the processes of cellular lipid accumulation and hepatic dysfunction, which are closely related to the development of NAFLD $(25,26)$. Figure 4 a demonstrates significant increases of more than twofold in the
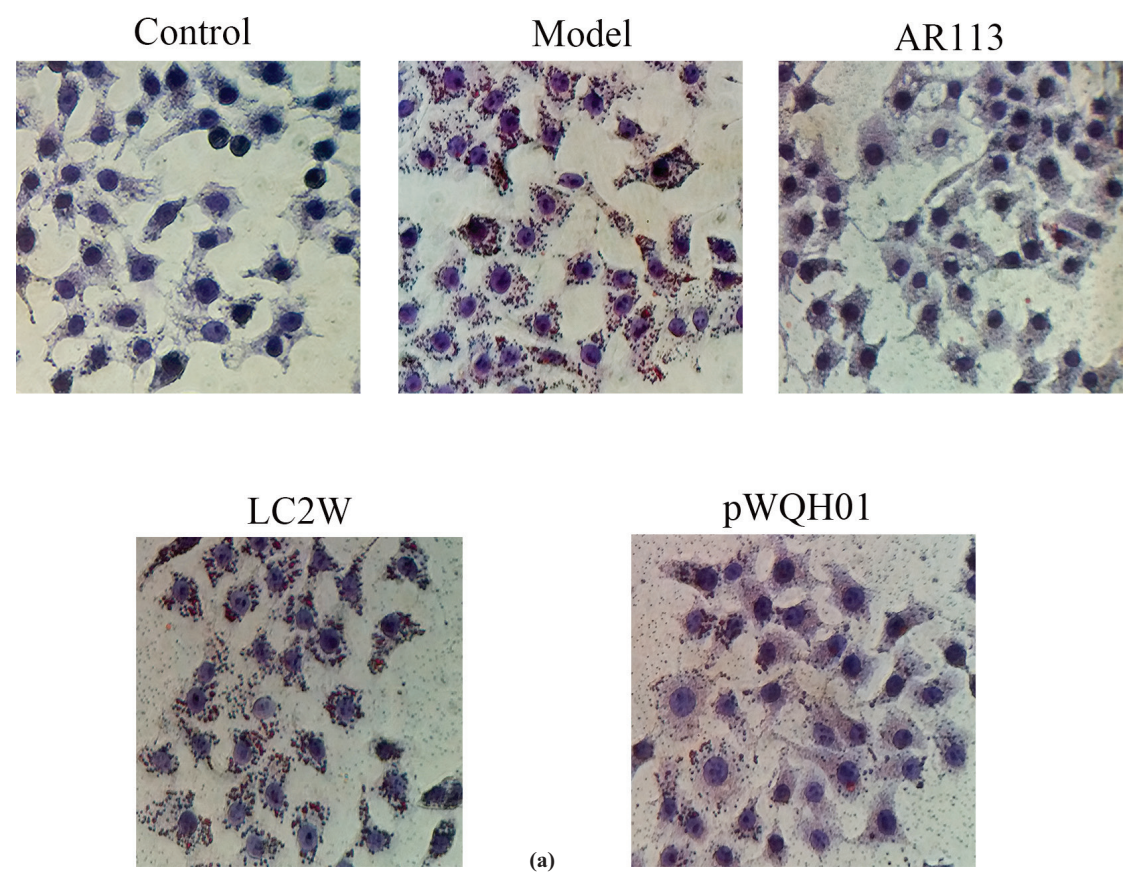

(a)
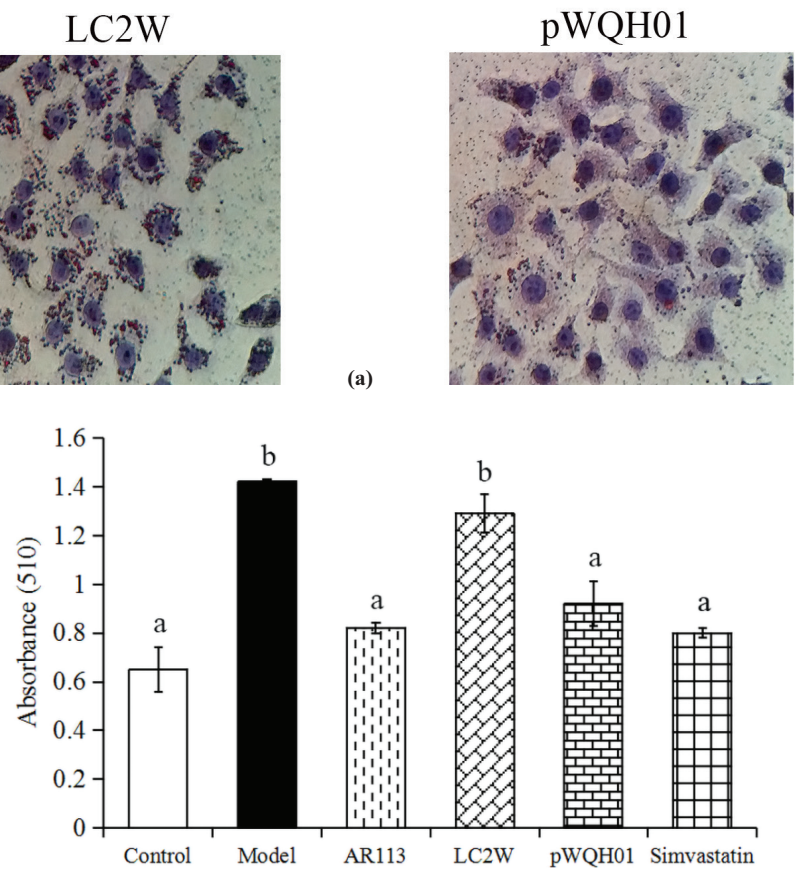

(b)

Fig. 2. The effects of probiotic strains on lipid accumulation and lipid contents in HepG2 cells. (a) After treatment with probiotic strains for $6 \mathrm{~h}$, lipids were stained with Oil Red O (400× magnification). (b) Spectrophotometric quantification of the total lipid contents by dissolving the stained oil droplets from HepG2 cells. Data represent the means \pm standard deviations $(n=3)$. Statistical differences between groups were determined by a one-way ANOVA. Different letters indicate statistically significant differences between groups, $P<0.05$. 
TC levels of OA- and cholesterol-treated cells relative to the untreated controls $(P<0.05)$. Promisingly, the cells treated with $L$. plantarum AR113 or $L$. casei $\mathrm{pWQH} 01$ resembled control cells, with significantly $(P<0.05)$ lower levels of TC accumulation than cells in the OA- and cholesterol-treated group, whereas L. casei LC2W-treated cells had levels similar to those of OA- and cholesterol-treated cells (Fig. 3a). These results are consistent with the above-described results of Oil Red O staining.

HMGCR is a rate-limiting enzyme in the metabolic pathway that produces cholesterol and other isoprenoids, and is a frequent target of cholesterol-lowering drugs. Figure $3 \mathrm{~b}$ shows that HMGCR expression increased significantly $(P<0.05)$ in the $\mathrm{OA}$ and cholesterol-treated group by almost fourfold. In contrast, cells treated with L. plantarum AR113 or L. casei $\mathrm{pWQH01}$ exhibited significantly $(P<0.05)$ decreased HMGCR expression, with levels below those detected in controls. Notably, HMGCR expression was much higher in $L$. case $i \mathrm{LC} 2 \mathrm{~W}$-treated cells than in cells treated with L. plantarum AR113 or L. casei pWQH01 or even the control group. These findings indicate that some probiotic strains may suppress HMGCR expression by altering $\mathrm{BSH}$ activity.

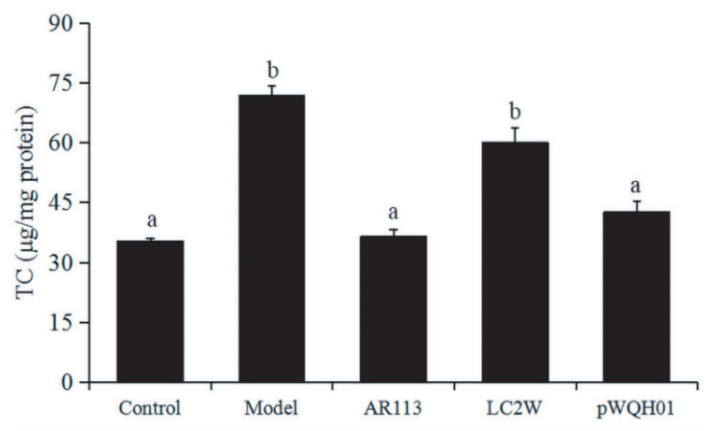

(a)

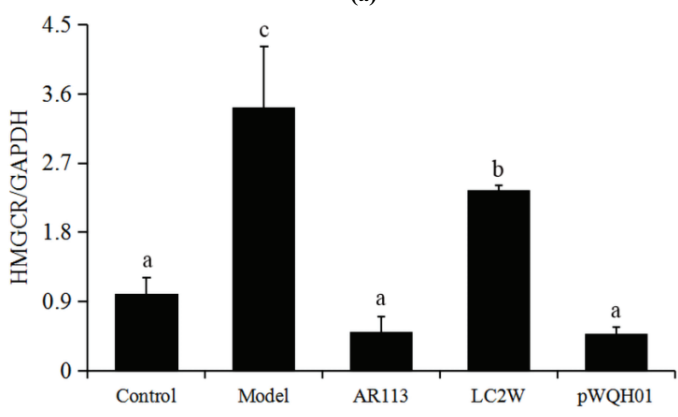

(b)

Fig. 3. The effects of probiotic strains on total cholesterol (TC) levels. (a) TC levels in HepG2 cells. (b) HMGCR mRNA levels. Data represent the means \pm standard deviations $(n=3)$. Statistical differences between groups were determined by a one-way ANOVA. Different letters indicate statistically significant differences between groups, $P<0.05$.
Probiotic strains modulate key lipid-regulating genes in HepG2 cells

To investigate the mechanism by which probiotic strains improve hepatic lipid accumulation in HepG2 cells, we monitored the mRNA expression of vital lipid-regulating genes in various treatment groups. Notably, we observed significantly higher expression of SREBP-1c, ACC, FAS and TNF $\alpha$ mRNA and significantly lower expression of AMPK and PPAR $\alpha$ mRNA in OA- and cholesterol-treated cells relative to control group cells (Fig. 4). However, exposure to L. plantarum AR113 or L. casei $\mathrm{pWQH01} \mathrm{sig-}$ nificantly reduced the expression of SREBP-1c, ACC, FAS and TNF $\alpha$ mRNA and significantly increased the expression of AMPK and PPAR $\alpha$ mRNA in OA- and cholesterol-treated cells. In contrast, no significant differences in the expression of AMPK, SREBP-1c, ACC and PPAR $\alpha$ mRNA were observed between $L$. casei LC2W and OA- and cholesterol-treated cells (Fig. 4). These indicate that BSH activity level plays an important role in the probiotic-mediated modulation of key lipid-regulating genes in HepG2 cells.

\section{Discussion}

NAFLD is a clinical pathological change characterised by the accumulation of triglycerides in hepatocytes and is among the most common causes of abnormal liver function tests (27). Recently, the incidence of NAFLD has increased significantly along with increases in the prevalence of dyslipidaemia, obesity and type 2 diabetes mellitus. According to one report, more than $10 \%$ of NAFLD patients progress to NASH, and some patients in the latter group eventually develop cirrhosis and hepatocellular carcinoma (28). Although some drugs have been developed to improve NAFLD, these induce many side effects (29). Therefore, new treatments for NAFLD/NASH are urgently needed.

In recent years, many studies have proposed the beneficial effects of probiotics on health $(30,31)$. Several lines of evidence have indicated the anti-obesity and hepatoprotective activities of probiotics (32). For example, probiotics can improve hepatic lipid metabolism and reduce serum triacylglycerol and total cholesterol (TC) levels in patients with NAFLD. For example, yogurt supplemented with L. acidophilus and Bifidobacterium spp. can reduce the serum levels of ALT, AST, TC and low-density lipoprotein cholesterol in patients with NAFLD (33). However, the roles of these probiotic strains and the molecular mechanism(s) underlying the alleviation of NAFLD pathogenesis remain poorly understood. In this study, we investigated the beneficial effects of L. plantarum AR113 and L. casei pWQH01, which exhibit high BSH activity, on cholesterol levels and hepatic lipid accumulation in vitro, as well as the mechanism underlying these effects. 

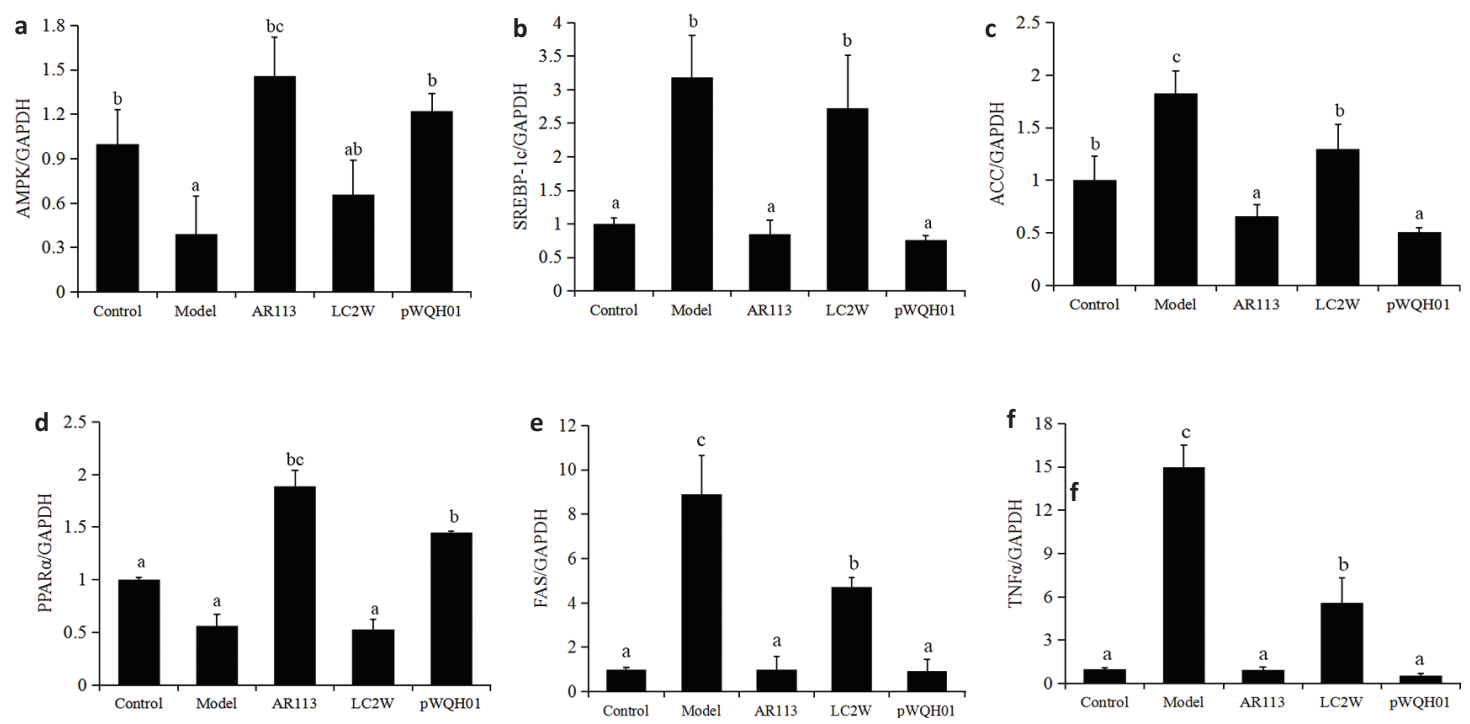

Fig. 4. The effects of probiotic strains on the mRNA expression of key lipid-regulating genes in HepG2 cells stimulated with oleic acid OA and cholesterol. (a) AMPK, (b) SREBP-1c, (c) ACC, (d) PPAR $\alpha$, (e) FAS and (f) TNF $\alpha$. Data represent the means \pm standard deviations $(n=3)$. Statistical differences between groups were determined by a one-way ANOVA. Different letters indicate statistically significant differences between groups, $P<0.05$.

Previous studies have demonstrated the considerable involvement of cholesterol metabolism in the development of hepatic lipid accumulation and cellular dysfunction, which are closely related to the development of $\operatorname{NAFLD~}(25,26)$. Our results demonstrated that the treatment of OA- and cholesterol-treated HepG2 cells with $L$. plantarum AR113 and L. casei pWQH01 led to significant decreases in the TC levels and HMGCR mRNA expression when compared with the OA and cholesterol treatment alone. In contrast, treatment with L. casei $\mathrm{LC} 2 \mathrm{~W}$ had no significant effects relative to OA and cholesterol treatment alone. As L. plantarum AR 113 and $L$. casei $\mathrm{pWQH} 01$ exhibit high BSH activity while L. casei $\mathrm{LC} 2 \mathrm{~W}$ has no BSH activity, our results suggest that BSH activity may be responsible for the beneficial effects of the former strains against NAFLD. These findings are consistent with those of previous studies $(34,35)$, which demonstrated that probiotics with BSH activity can reduce cholesterol levels and serum lipid parameters (TC and low-density lipoprotein cholesterol levels) $(34,35)$. Our Oil Red O staining examination revealed a marked intracellular accumulation of lipids in the HepG2 cell model of NAFLD (OA- and cholesterol-treated cells). The total lipid and TC levels were lower in the L. plantarum AR113 and L. casei $\mathrm{pWQH} 01$ groups than in the OA- and cholesterol-treated and $L$. case $i \mathrm{LC} 2 \mathrm{~W}$-treated groups. These results show that exposure to L. plantarum AR113 and L. casei pWQH01 can significantly prevent hepatic lipid accumulation by reducing cholesterol synthesis in a BSH-dependent manner.
To further investigate the mechanism by which L. plantarum $\mathrm{AR} 113$ and L. casei $\mathrm{pWQH} 01$ ameliorate hepatic lipid accumulation, we quantitatively analysed the expression of key genes involved in fatty acid synthesis and metabolism using qRT-PCR. Steatosis is caused primarily by an increased flux of fatty acids to the liver, which is consequent to the high availability of plasma FFAs relative to the peripheral oxidative requirements (20). AMPK is an energy sensor that regulates hepatic lipogenesis and may thus be a therapeutic target in the treatment of fatty liver disease (36). Once activated, AMPK inhibits ACC expression by down-regulating SREBP-1c and thus attenuates hepatic steatosis (37). SREBP-1c and FAS are also involved in fatty acid synthesis and the development and pathogenesis of NAFLD (38). In our study, OA- and cholesterol-treated cells exhibited significant increases in the expression of SREBP-1c, ACC and FAS mRNA that paralleled a decrease in the expression of AMPK mRNA. This finding indicates a negative feedback loop that regulates SREBP-1c expression via AMPK upon exposure to OA and cholesterol. Compared with the untreated model cells, cells treated with $L$. plantarum AR113 and L. casei $\mathrm{pWQH01}$ exhibited significant decreases in the expression of SREBP-1c, ACC and FAS mRNA and a significantly increase in AMPK mRNA, whereas no significant changes were observed upon exposure to $L$. casei LC2W. In other words, L. plantarum AR113 and L. casei pWQH01 appear to reduce hepatic lipid accumulation by targeting AMPK-mediated fatty acid synthesis and thus inhibiting SREBP-1c, ACC and FAS expression in a BSH-dependent manner. 
PPAR $\alpha$ induces the expression of various target genes involved in the uptake, transportation and $\beta$-oxidation of fatty acids (39). PPAR $\alpha$ also augments fatty acid catabolism and thus may protect against liver fat deposition (40). In this study, OA- and cholesterol-induced steatosis led to a decrease in PPAR $\alpha$ expression in HepG2 cells, suggesting that this receptor may play a pathogenic role in NAFLD. Our results demonstrated an increase in the expression of PPAR $\alpha$ after treatment with L. plantarum AR 113 or L. casei pWQH01. The results may also indicate that $L$. plantarum AR113 and $L$. casei $\mathrm{pWQH} 01$ can exploit BSH to reduce liver fat deposition by decreasing lipid peroxidation, which may be mediated by enhanced PPAR $\alpha$ expression.

Several studies have observed the overexpression of TNF $\alpha$ mRNA in both the liver and adipose tissues of severely obese patients with NASH (41). An elevated $\mathrm{TNF} \alpha$ level plays a key role in the pathogenesis and disease progression of NAFLD (42). Previous studies have reported that the treatment of HepG2 cells with FFA may upregulate the expression of TNF $\alpha$ mRNA (43). In our study, we confirmed that OA treatment significantly upregulated the expression of TNF $\alpha$ mRNA in HepG2 cells, whereas exposure to $L$. plantarum AR113 and L. casei $\mathrm{pWQH} 01$ significantly decreased this expression. Our data further support a pathogenic role for hepatocyte-derived TNF $\alpha$ in NAFLD. However, it remains to be determined whether the increased TNF $\alpha$ in NAFLD is derived from hepatocytes or other inflammatory cells.

\section{Conclusions}

Taken together, our data suggest L. plantarum AR 113 and L. casei $\mathrm{pWQH01,} \mathrm{which} \mathrm{both} \mathrm{exhibit} \mathrm{high} \mathrm{BSH} \mathrm{activity,}$ might protect the liver from NAFLD and decrease hepatic lipid accumulation by reducing fatty acid synthesis. These beneficial effects may be mediated via the modulation of cholesterol synthesis and lipid-regulating gene expression in a BSH-dependent manner. L. casei $\mathrm{LC} 2 \mathrm{~W}$, which lacks BSH activity, cannot mediate these effects. Our results indicate that BSH plays an important role in the beneficial effects of Lactobacillus strains in the treatment of NAFLD. In the future, probiotics with high BSH activity should be considered as potentially beneficial for the treatment of NAFLD.

\section{Acknowledgements}

This work was supported by the National Natural Science Foundation of China (No. 31972056) and Shanghai Agriculture Applied Technology Development Program, China (Grant No. 2019-02-08-00-07-F01152).

\section{Conflicts of interest and funding}

There are no conflicts of interest to declare. The authors have not received any funding or benefits from industry or elsewhere to conduct this study.

\section{References}

1. Satapathy SK, Sanyal AJ. Epidemiology and natural history of nonalcoholic fatty liver disease. Semin Liver Dis 2015; 35(3): 221-35. doi: 10.1055/s-0035-1562943

2. Dinani A, Sanyal A. Nonalcoholic fatty liver disease: implications for cardiovascular risk. Cardiovasc Endocrinol 2017; 6(2): 62-72. doi: 10.1097/XCE.0000000000000126

3. Tiniakos DG, Vos MB, Brunt EM. Nonalcoholic fatty liver disease: pathology and pathogenesis. Annu Rev Pathol 2010; 5: 145-71. doi: 10.1146/annurev-pathol-121808-102132

4. Bellentani S, Scaglioni F, Marino M, Bedogni G. Epidemiology of non-alcoholic fatty liver disease. Dig Dis 2010; 28(1): 155-61. doi: $10.1159 / 000282080$

5. Arguello G, Balboa E, Arrese M, Zanlungo S. Recent insights on the role of cholesterol in non-alcoholic fatty liver disease. Biochim Biophys Acta 2015; 1852(9): 1765-78. doi: 10.1016/j. bbadis.2015.05.015

6. Min HK, Kapoor A, Fuchs M, Mirshahi F, Zhou H, Maher J, et al. Increased hepatic synthesis and dysregulation of cholesterol metabolism is associated with the severity of nonalcoholic fatty liver disease. Cell Metab 2012; 15(5): 665-74. doi: 10.1016/j. cmet.2012.04.004

7. Rotman Y, Sanyal AJ. Current and upcoming pharmacotherapy for non-alcoholic fatty liver disease. Gut 2017; 66(1): 180-90. doi: 10.1136/gutjnl-2016-312431

8. Chandrasekaran VRM, Hsu DZ, Chien SP, Liu MY. Co-exposure of arsenic and iron causes hepatic injury: a tale of two hits. Epidemiology 2009; 20(6): S125. doi: 10.1097/01.ede.0000362432. 97462.c5

9. Li WP, Chen XG, Lin MZ, Huang DY. Up-regulated HOTAIR induced by fatty acids inhibits PTEN expression and increases triglycerides accumulation in HepG2 cells. Food Nutr Res 2017; 61: 1412794. doi: 10.1080/16546628.2017.1412794

10. Araya J, Rodrigo R, Videla LA, Thielemann L, Orellana M, Pettinelli $\mathrm{P}$, et al. Increase in long-chain polyunsaturated fatty acid n-6/n-3 ratio in relation to hepatic steatosis in patients with non-alcoholic fatty liver disease. Clin Sci (Lond) 2004; 106(6): 635-43. doi: 10.1042/CS20030326

11. Janorkar AV, King KR, Megeed Z, Yarmush ML. Development of an in vitro cell culture model of hepatic steatosis using hepatocyte-derived reporter cells. Biotechnol Bioeng 2009; 102(5): 1466-74. doi: 10.1002/bit.22191

12. Hill C, Guarner F, Reid G, Gibson GR, Merenstein DJ, Pot B, et al. Expert consensus document: the international scientific association for probiotics and prebiotics consensus statement on the scope and appropriate use of the term probiotic. Nat Rev Gastroenterol Hepatol 2014; 11(8): 506-14. doi: 10.1038/nrgastro.2014.66

13. Korpela K, Salonen A, Vepsäläinen O, Suomalainen M, Kolmeder C, Varjosalo M, et al. Probiotic supplementation restores normal microbiota composition and function in antibiotic-treated and in caesarean-born infants. Microbiome 2018; 6(1): 182. doi: 10.1186/s40168-018-0567-4

14. Hidalgo-Cantabrana C, Delgado S, Ruiz L, Ruas-Madiedo P, Sánchez B, Margolles A. Bifidobacteria and their healthpromoting effects. Microbiol Spectr 2017; 5(3). doi: 10.1128/ microbiolspec.BAD-0010-2016

15. Alisi A, Bedogni G, Baviera G, Giorgio V, Porro E, Paris C, et al. Randomised clinical trial: the beneficial effects of vsl\#3 in obese children with non-alcoholic steatohepatitis. Aliment Pharmacol Ther 2014; 39(11): 1276-85. doi: 10.1111/apt.12758

16. Mouzaki M, Bandsma R. Targeting the gut microbiota for the treatment of non-alcoholic fatty liver disease. Curr Drug 
Targets 2015; 16(12): 1324-31. doi: 10.2174/1389450116666150 416120351

17. Foley MH, O'Flaherty S, Barrangou R, Theriot CM. Bile salt hydrolases: gatekeepers of bile acid metabolism and host-microbiome crosstalk in the gastrointestinal tract. PLoS Pathog 2019; 15(3): e1007581. doi: 10.1371/journal. ppat. 1007581

18. Wang G, Zhang Y, Song X, Xia Y, Lai PF, Ai L. Lactobacillus casei $\mathrm{LC} 2 \mathrm{~W}$ can inhibit the colonization of Escherichia coli O157:H7 in vivo and reduce the severity of colitis. Food Funct 2019, 10: 5843-5852. doi: 10.1039/C9FO01390C.

19. Cousin SP, Hügl SR, Wrede CE, Kajio H, Myers MG Jr, Rhodes CJ. Free fatty acid-induced inhibition of glucose and insulin-like growth factor I-induced deoxyribonucleic acid synthesis in the pancreatic beta-cell line INS-1. Endocrinology 2001; 142(1): 229-40. doi: 10.1210/endo.142.1.7863

20. Li X, Wang R, Zhou N, Wang X, Liu Q, Bai Y, et al. Quercetin improves insulin resistance and hepatic lipid accumulation in vitro in a NAFLD cell model. Biomed Rep 2013; 1(1): 71-76. doi: 10.3892/br.2012.27

21. Horáčková Š, Plocková M, Demnerová K. Importance of microbial defence systems to bile salts and mechanisms of serum cholesterol reduction. Biotechnol Adv 2018; 36(3): 682-690. doi: 10.1016/j.biotechadv.2017.12.005

22. Livak KJ, Schmittgen TD. Analysis of relative gene expression data using real-time quantitative PCR and the 2(-Delta Delta C(T)) Method. Methods 2001; 25(4): 402-8. doi: 10.1006/meth. 2001.1262

23. Hwang YJ, Lee EJ, Kim HR, Hwang KA. Nf-kb-targeted antiinflammatory activity of Prunella vulgaris var. lilacina in macrophages raw 264.7. Int J Mol Sci 2013; 14(11): 21489-503. doi: $10.3390 /$ ijms 141121489

24. Hostanska K, Suter A, Melzer J, Saller R. Evaluation of cell death caused by an ethanolic extract of Serenoae repentis fructus (prostasan) on human carcinoma cell lines. Anticancer Res 2007; 27(2): 873-81. doi: 0250-7005/2007 \$2.00+.40

25. de Boer JF, Schonewille M, Boesjes M, Wolters H, Bloks VW, Bos $\mathrm{T}$, et al. Intestinal farnesoid $\mathrm{X}$ receptor controls transintestinal cholesterol excretion in mice. Gastroenterology 2017; 152(5): 1126-38. doi: 10.1053/j.gastro.2016.12.037

26. Temel R. Hepatic or intestinal ABCG5 and ABCG8 are sufficient to block the development of sitosterolemia. J Lipid Res 2015; 56(2): 201-2. doi: 10.1194/jlr.C056945

27. Paschos P, Paletas K. Non-alcoholic fatty liver disease and metabolic syndrome. Hippokratia 2009; 13(1): 9-19. Available from: https://www.ncbi.nlm.nih.gov/pmc/articles/PMC2633261/

28. Mavrogiannaki AN, Migdalis IN. Nonalcoholic fatty liver disease, diabetes mellitus and cardiovascular disease: newer data. Int J Endocrinol 2013; 2013: 450639. doi: 10.1155/ 2013/450639

29. Pferschy-Wenzig EM, Atanasov AG, Malainer C, Noha SM, Kunert O, Schuster D, et al. Identification of isosilybin a from milk thistle seeds as an agonist of peroxisome proliferator-activated receptor gamma. J Nat Prod 2014; 77(4): 842-7. doi: $10.1021 / \mathrm{np} 400943 \mathrm{~b}$

30. Wang G, Zhang Y, Song X, Xia Y, Lai PF, Ai L. Lactobacillus casei $\mathrm{LC} 2 \mathrm{~W}$ can inhibit the colonization of Escherichia coli O157:H7 in vivo and reduce the severity of colitis. Food Funct 2019; 10: 5843-52. doi: 10.1039/C9FO01390C

31. Han KJ, Lee NK, Park H, Paik HD. Anticancer and antiinflammatory activity of probiotic Lactococcus lactis NK34.
J Microbiol Biotechnol 2015; 25(10): 1697-701. doi: 10.4014/ jmb. 1503.03033

32. Kadooka Y, Sato M, Imaizumi K, Ogawa A, Ikuyama K, Akai $\mathrm{Y}$, et al. Regulation of abdominal adiposity by probiotics (Lactobacillus gasseri SBT2055) in adults with obese tendencies in a randomized controlled trial. Eur J Clin Nutr 2010; 64(6): 636-43. doi: 10.1038/ejen.2010.19

33. Malaguarnera M, Vacante M, Antic T, Giordano M, Chisari G, Acquaviva R, et al. Bifidobacterium longum with fructo-oligosaccharides in patients with non-alcoholic steatohepatitis. Dig Dis Sci 2012; 57(2): 545-53. doi: 10.1007/s10620-011-1887-4

34. Guo CF, Zhang S, Yuan YH, Li JY, Yue TL. Bile salt hydrolase and s-layer protein are the key factors affecting the hypocholesterolemic activity of Lactobacillus casei-fermented milk in Hamsters. Mol Nutr Food Res 2018; 62(24): e1800728. doi: 10.1002/ mnfr.201800728

35. Le B, Yang SH. Identification of a novel potential probiotic Lactobacillus plantarum FB003 isolated from salted-fermented shrimp and its effect on cholesterol absorption by regulation of NPC1L1 and PPAR $\alpha$. Probiotics Antimicrob Proteins. 2019; 11(3): 785-93. doi: 10.1007/s12602-018-9469-9

36. Long YC, Zierath JR. Amp-activated protein kinase signaling in metabolic regulation. J Clin Invest 2006; 116(7): 1776-83. doi: $10.1172 / \mathrm{JCI} 29044$

37. Li Y, Xu S, Mihaylova MM, Zheng B, Hou X, Jiang B, et al. AMPK phosphorylates and inhibits SREBP activity to attenuate hepatic steatosis and atherosclerosis in diet-induced insulin-resistant mice. Cell Metab 2011; 13(4): 376-88. doi: 10.1016/j.cmet.2011.03.009

38. Horton JD, Bashmakov Y, Shimomura I, Shimano H. Regulation of sterol regulatory element binding proteins in livers of fasted and refed mice. Proc Natl Acad Sci U S A 1998; 95(11): 5987-92. doi: 10.1073/pnas.95.11.5987

39. Desvergne B, Wahli W. Peroxisome proliferator-activated receptors: nuclear control of metabolism. Endocr Rev 1999; 20(5): 649-88. doi: 10.1210/edrv.20.5.0380

40. Svegliati-Baroni G, Candelaresi C, Saccomanno S, Ferretti G, Bachetti T, Marzioni M, et al. A model of insulin resistance and nonalcoholicsteatohepatitisin rats:role of peroxisome proliferatoractivated receptor- $\alpha$ and n-3 polyunsaturated fatty acid treatment on liver injury. Am J Pathol 2006; 169(3): 846-60. doi: 10.2353/ ajpath.2006.050953

41. Crespo J, Cayón A, Fernández-Gil P, Hernández-Guerra M, Mayorga M, Domínguez-Díez A, et al. Gene expression of tumor necrosis factor alpha and TNF-receptors, p55 and p75, in nonalcoholic steatohepatitis patients. Hepatology 2001; 34(6): 1158-63. doi: 10.1053/jhep.2001.29628

42. Wong VW, Hui AY, Tsang SW, Chan JL, Tse AM, Chan KF, et al. Metabolic and adipokine profile of Chinese patients with nonalcoholic fatty liver disease. Clin Gastroenterol Hepatol 2006; 4(9): 1154-61. doi: 10.1016/j.cgh.2006.06.011

43. Cui W, Chen SL, Hu KQ. Quantification and mechanisms of oleic acid-induced steatosis in HepG2 cells. Am J Transl Res. 2010; 2(1): 95-104: PMCID: PMC2826826. Available from: www.ajtr.org/AJTR910003

\footnotetext{
*Lianzhong Ai

University of Shanghai for Science and Technology

516 Jungong Road

Shanghai 200093, China

Email: ailianzhong@hotmail.com
} 